\title{
Scaling properties of planetary calderas and terrestrial volcanic eruptions
}

\author{
L. Sanchez ${ }^{1}$ and R. Shcherbakov ${ }^{1,2}$ \\ ${ }^{1}$ Department of Earth Sciences, University of Western Ontario, London, Ontario, N6A 5B7, Canada \\ ${ }^{2}$ Department of Physics and Astronomy, University of Western Ontario, London, Ontario, N6A 3K7, Canada
}

Correspondence to: L. Sanchez (lsanche@uwo.ca) and R. Shcherbakov (rshcherb@uwo.ca)

Received: 26 June 2012 - Revised: 4 October 2012 - Accepted: 12 October 2012 - Published: 6 November 2012

\begin{abstract}
Volcanism plays an important role in transporting internal heat of planetary bodies to their surface. Therefore, volcanoes are a manifestation of the planet's past and present internal dynamics. Volcanic eruptions as well as caldera forming processes are the direct manifestation of complex interactions between the rising magma and the surrounding host rock in the crust of terrestrial planetary bodies. Attempts have been made to compare volcanic landforms throughout the solar system. Different stochastic models have been proposed to describe the temporal sequences of eruptions on individual or groups of volcanoes. However, comprehensive understanding of the physical mechanisms responsible for volcano formation and eruption and more specifically caldera formation remains elusive. In this work, we propose a scaling law to quantify the distribution of caldera sizes on Earth, Mars, Venus, and Io, as well as the distribution of calderas on Earth depending on their surrounding crustal properties. We also apply the same scaling analysis to the distribution of interevent times between eruptions for volcanoes that have the largest eruptive history as well as groups of volcanoes on Earth. We find that when rescaled with their respective sample averages, the distributions considered show a similar functional form. This result implies that similar processes are responsible for caldera formation throughout the solar system and for different crustal settings on Earth. This result emphasizes the importance of comparative planetology to understand planetary volcanism. Similarly, the processes responsible for volcanic eruptions are independent of the type of volcanism or geographical location.
\end{abstract}

\section{Introduction}

The formation of volcanoes and their corresponding eruption dynamics is a complex phenomenon and a major natural hazard on Earth. Planetary exploration has shown that volcanism is or was operational on other solar system bodies such as Mars, Venus, Mercury, the Moon, Io, and several other planetary satellites (Wilson, 2009; Prockter et al., 2010). Past studies have attempted to compare volcanic structures on Earth to similar features on other planetary bodies with some success. Similarities have been found between Martian cones and cinder cones on Earth (Frey and Jarosewich, 1982) and also between small Venusian domes, shields and cones and terrestrial sea floor volcanoes (Smith, 1996; Bulmer and Wilson, 1999). The importance of considering calderas in comparative planetology was emphasized (Wood, 1984).

A planetary definition of a caldera is a multi-kilometer wide, quasi-circular depression, not of impact origin, formed in volcanic terrain by the collapse of the volcanic edifice into a partially drained magma chamber (Lipman, 2000). An equivalent volcanic feature, which is found on Mars, Venus, and Io, is described as an irregular volcanic crater with scalloped edges is a patera. The general consensus in the interpretation of paterae on Venus and Mars is that they are analogs for calderas (Sigurdsson et al., 2000). This argument has been supported by the comparison of the distributions and morphology of paterae on Io to calderas on Earth and paterae on Mars and Venus (Radebaugh et al., 2001). The increase in space remote sensing data has allowed planetary calderas to be studied and compared throughout the solar system in order to understand their formation processes. The geomorphology of calderas on Earth, Mars and Venus has been analyzed to better understand basaltic volcanoes 
using comparative planetology (Mouginis-Mark and Rowland, 2001). An important characteristic of calderas/paterae is their diameter and area which are related to the size of the underlying magma chamber and can be used as a proxy for estimating the potential of volcanic eruptions (Lipman, 2000). The distribution of magma chamber sizes for planetary bodies is directly related to the crustal thickness and the properties of magma material such as density, concentration of volatiles, etc. (Mouginis-Mark and Rowland, 2001). As a result, constraining the volcano forming and eruption processes would lead to a better understanding of the dynamical evolution of planetary interiors (Sobradelo et al., 2010). Several numerical and analog models have been proposed to analyze and understand caldera-forming eruptions in the solar system (Kieffer, 1995; Acocella, 2007, and references therein).

Statistical studies have also been carried out to analyze calderas on Earth. Sobradelo et al. (2010) performed ANOVA (analysis of variance) on the distribution of caldera areas in order to relate the size of calderas with various geodynamical settings. The analysis was successful at determining three distinct geodynamical environments that host small, medium and large calderas. Hughes and Mahood (2011) studied the spatial distribution of calderas in arc settings and were able to correlate the spatial distribution of calderas with the tectonic properties of arcs.

Another manifestation of complex magmatic processes within the crust is the occurrence of volcanic eruptions. Statistical analysis is a powerful tool that can be used to identify patterns and correlations in the occurrence of volcanic eruptions. Numerous statistical analyses have been performed on eruption time series on Earth. The earliest ones used Markov models to reproduce distribution of repose times of volcanoes in Hawaii (Wickman, 1966). Since then, different stochastic models have been suggested to quantify the statistical aspects of eruptions. De la Cruz-Reyna (1991) proposed a Poisson model to describe the global distribution of large volcanic eruptions. Ho (1991) and Bebbington and Lai (1996) proposed, respectively, a non-homogeneous Poisson model and a Weibull renewal model that they tested using the occurrence of eruptions on Aso, Mount Etna, Mount St. Helens, Kilauea and Yake-Dake volcanoes. The nonhomogeneous Poisson model was used to fit successfully the distribution of flank eruptions for Mount Etna (Salvi et al., 2006). Mendoza-Rosas and De la Cruz-Reyna (2009) tested a mixture of exponential distributions to model the occurrence of volcanic events in Mexico with some success. The temporal structure of global sequences of explosive eruptions in Kamchatka was analyzed and self-similar clustering and episodicity were observed (Gusev et al., 2003). Clustering was also observed on the onsets of volcanic eruptions and their statistical behavior was modeled using a trend renewal process (Bebbington, 2010). In order to predict the repose time of future extreme volcanic eruptions, Pyle (1998) used rank-ordering power-law statistics on data sets from the
Taupo volcano in New Zealand. Deligne et al. (2010) examined the frequency-magnitude statistics of historical recurrence rates of large explosive eruptions using extreme value theory.

An alternative approach to study volcanic processes is to look at the phenomenon as a whole in order to develop a general framework applicable to all the volcanoes on Earth and in the solar system, independent of the volcano's crustal surrounding and geographical location. This type of global approach has been proven successful at defining scaling laws for the occurrence of other natural hazards such as forest fires (Corral et al., 2008), earthquakes (Corral, 2003; Shcherbakov et al., 2005), solar flares (Baiesi et al., 2006) and tropical cyclones (Corral et al., 2010). This approach was also successfully used in analyzing the fracture of rocks (Davidsen et al., 2007). Despite their complexity, volcanic processes can also be approximated by a point process in space and time. Using this approach, several studies were undertaken to analyze global eruption time series. Gusev (2008) observed selfsimilar clustering in time and size for eruptions. It was also observed that large eruptions tend to occur during the most volcanically active periods. These characteristics of global volcanic activity lead to the conclusion that a global mechanism was responsible for the time/size clustering. Marzocchi and Zaccarelli (2006) observed two different regimes concerning interevent times: short times are clustered and can be explained by an open conduit system, while long interevent times show random behavior, which can be characterized by a Poisson process and explained by a closed conduit system.

In this work, we investigated the universal properties of the size distribution of calderas/paterae in the solar system. Particularly, we considered four planetary bodies: the Earth, Mars, Venus, and Io. We showed that these distributions of caldera diameters and areas can be approximated by a universal functional form when they were rescaled with the corresponding sample averages. We also analyzed the distribution of interevent times between successive eruptions of volcanoes around the world. Those distributions also displayed a universal behavior when rescaled with the corresponding mean interevent times. This approach allowed us to conclude that the caldera formation was governed by similar processes throughout the solar system. The temporal characteristics of eruptions processes on Earth were also quite similar among different volcanoes and tectonic settings.

The paper is organized as follows. In the next section we present the analysis of the planetary caldera data. In Sect. 3 we study the temporal scaling properties of the eruption time series considering 199 volcanoes around the world. In the last section, we discuss the obtained results concerning their implications to the volcanic processes in the solar system. 
Table 1. Summary of the data used for the planetary caldera analysis. $D_{n}$ is the mean diameter and $A_{n}$ is the mean caldera area computed from the minimum and maximum diameters assuming an elliptic shape for each planetary body, except for the Earth where some caldera areas are estimated using the actual shape (Geyer and Marti, 2008). The errors are given at $95 \%$ confidence intervals.

\begin{tabular}{lrrr}
\hline Planetary body & \#calderas & $D_{n}(\mathrm{~km})$ & $A_{n}\left(\mathrm{~km}^{2}\right)$ \\
\hline Earth & 386 & $12.6 \pm 1.1$ & $205.6 \pm 48.4$ \\
Venus & 83 & $62.3 \pm 6.8$ & $3741.0 \pm 947.0$ \\
Mars & 19 & $102.0 \pm 44.8$ & $14615.0 \pm 1285.0$ \\
Io & 144 & $72.7 \pm 7.4$ & $5715.0 \pm 1430.0$ \\
Continental crust & 209 & & $289.7 \pm 42.3$ \\
Oceanic crust & 44 & & $111.3 \pm 57.0$ \\
Transitional crust & 85 & & $98.5 \pm 12.4$ \\
\hline
\end{tabular}

\section{Planetary caldera analysis}

\subsection{Solar system bodies}

As previously stated, paterae are volcanic features present on Mars, Venus and Io and are analogs of calderas on Earth. For our analysis, we consider that paterae are analogs of volcanic calderas, and therefore we use the term caldera in the rest of the text. We extracted caldera size data from the Collapse Caldera Database (CCDB) (Geyer and Marti, 2008) for the Earth from the Magellan Venus volcanic feature catalog (Crumpler et al., 1997), and from the USGS astrogeology research program (USGS, last consulted in April 2012). For all the databases mentioned, we were able to obtain diameter values in kilometers. For the caldera diameters on Earth and Venus, two measurements (minimum and maximum diameter) were reported in the catalogs, whereas for Mars and Io only one measurement was reported assuming that calderas were circular in shape. In our analysis, we used the mean diameters for each caldera and also computed the areas of each caldera by assuming that they can be approximated by an ellipse, except for the Earth where some actual areas of calderas were reported in the CCDB (see Table 1). When the minimum and the maximum diameters were given in the catalog, we computed the average value between the two.

To analyze the statistics of caldera sizes, we constructed the probability density functions of caldera diameters and areas for each planetary body. Because multiple scales were involved (from a few kilometers to $10^{3} \mathrm{~km}$ and from hundreds of square meters to $10^{3} \mathrm{~km}^{2}$ ), we used logarithmically increasing bins over which we calculated the probability densities. We defined as $x_{n}$ the coordinate of the right end of the $n$-th bin $(n=0,1,2, \ldots)$. The coordinate $x_{n+1}$ of the $(n+1)$ th bin was calculated recursively, $x_{n+1}=b x_{n}$, where $b$ was a bin factor. The length of the $n$-th bin was given by $\Delta x_{n}=$ $x_{0}\left(b^{n}-b^{n-1}\right)$. In our analysis the number of bins was chosen for each distribution and the corresponding bin factors $b$ were computed. The normalized probability density functions were calculated by dividing the number of occurrences

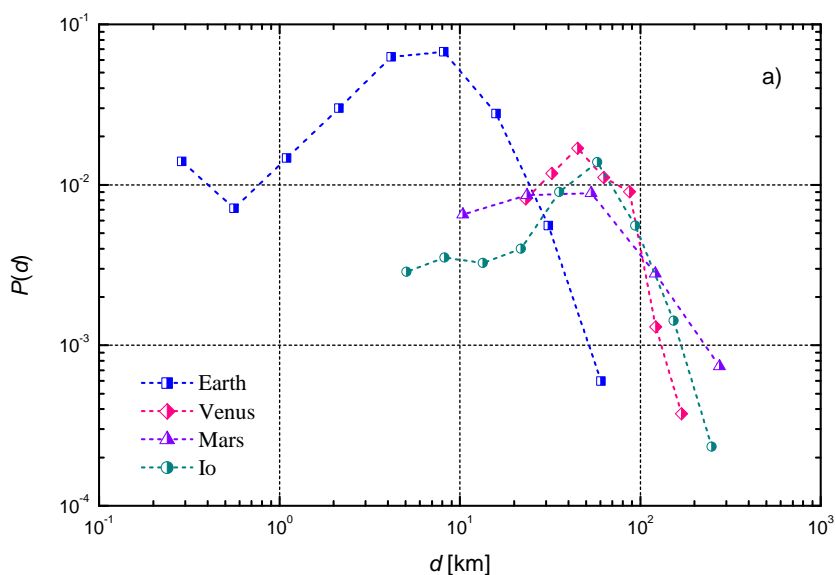

(a)

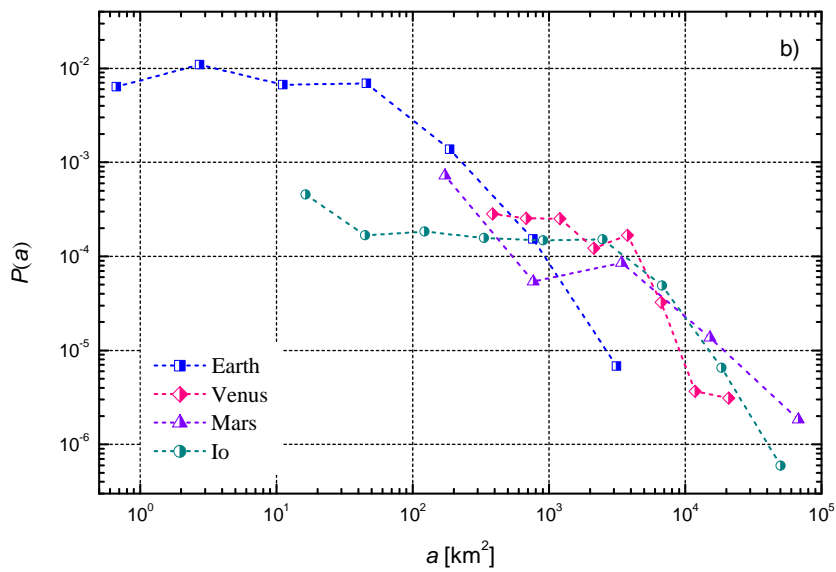

(b)

Fig. 1. Distributions of caldera sizes for the Earth, Venus, Mars and Io. (a) Caldera diameters in $\mathrm{km}$, (b) Caldera areas in $\mathrm{km}^{2}$.

within each bin by the corresponding bin length and the total number of data points considered. Due to the limited number of data points available for the Martian calderas, the distribution contained only a few points. The distributions of diameters and areas for each planetary body considered are presented in Fig. 1.

The constructed distributions display a similar behavior for all four bodies. There is a general power-law trend for large calderas and rollover and plateau for small diameters/areas. This change in the distributions can be attributed to different types of calderas present on those planetary bodies (Lipman, 2000). Large calderas are usually formed through a plate (piston) or downsag subsidence, while small calderas frequently have funnel geometry and are associated with explosive eruptions through the central vent and a subsequent subsidence (Lipman, 2000; Acocella, 2007). It is evident from Fig. 1 that the distribution of caldera sizes on Earth is shifted to the left, indicating the presence of smaller caldera sizes compared to the other planetary bodies. An explanation for this discrepancy can be related to the different 


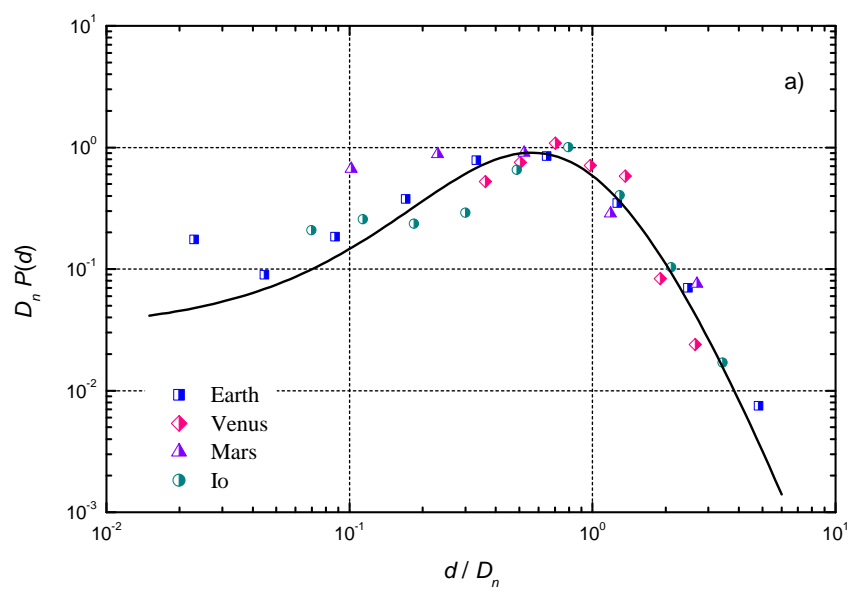

(a)

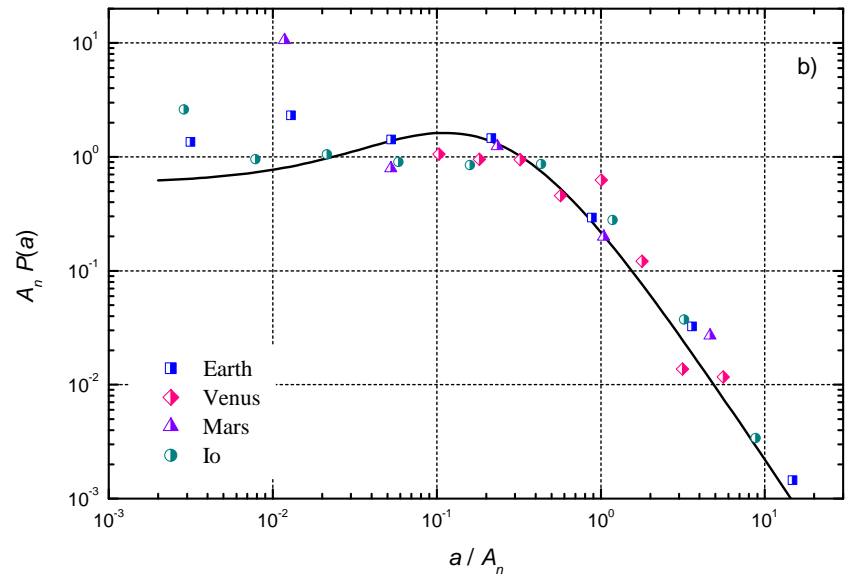

(b)

Fig. 2. Rescaled caldera/paterae distributions for Earth, Mars, Venus and Io according to Eq. (1): (a) rescaled diameters, with the mean diameter $D_{n}$ computed for each planetary body (Table 1 ). The solid curve is a fit to the rescaled distributions and is given by the GEV distribution, Eq. (2), with $\theta=0.22 \pm 0.06, \mu=0.64 \pm 0.04$, and $\sigma=$ $0.42 \pm 0.03$; (b) rescaled areas, with the mean area $A_{n}$ computed for each planetary body (Table 1 ). The solid curve is a fit to the rescaled distributions and is given by the GEV distribution, Eq. (2), with $\theta=0.84 \pm 0.11, \mu=0.26 \pm 0.03$, and $\sigma=0.30 \pm 0.03$.

caldera forming processes operating on those planetary bodies: on Earth, most calderas are formed around subduction zones and after large explosive (silicic) eruptions. On Mars, for example, there is no evidence of subduction zones and most volcanism is thought to be basaltic (non silicic). These factors can affect the size of the resulting calderas (Wood, 1984).

In order to compare the distributions, we performed a scaling analysis. We first calculated the sample averages $D_{n}$ and $A_{n}$ of caldera diameters and areas, where $n$ enumerated a planetary body (the Earth, Mars, Venus, and Io). We then rescaled the individual probability density functions, $P(d)$ and $P(a)$, according to
Table 2. The maximum values of the $\log$-likelihood function $(\ln L)$ and the corresponding values of the Akaike Information Criterion (AIC) for the model distribution functions considered to fit the rescaled distributions given in Fig. 2a.

\begin{tabular}{lccc}
\hline Distribution & $k$ & $\ln L$ & AIC \\
\hline Generalized Pareto & 2 & -627.15 & 1258.29 \\
Log-normal & 2 & -539.72 & 1083.45 \\
Generalized Extreme Value & 3 & -521.85 & 1049.70 \\
\hline
\end{tabular}

$\bar{x} P(x)=f\left(\frac{x}{\bar{x}}\right)$,

where $x \equiv d$ and $\bar{x} \equiv D_{n}$ for the diameters, $x \equiv a$ and $\bar{x} \equiv$ $A_{n}$ for the caldera areas, and $f(y)$ is a scaling function. Figure 2 shows the resulting plots of the rescaled distributions. After rescaling the distributions using the mean diameter/area according to Eq. (1), we observed a good collapse of the data into a single functional form. In order to model this functional form, we tested 3 different heavy tailed distributions: the generalized Pareto distribution, the log-normal distribution, and the generalized extreme value distribution (GEV). We estimated the parameters for each of them using the maximum likelihood method, which is not affected by the varying bin sizes of the distributions. The maximum likelihood estimation was done using the Matlab software. In order to determine best fitting distribution to model the scaling function $f(y)$, we computed the Akaike information criterion (AIC), which provides a relative goodness of fit, taking into account the number of parameters in each model (Akaike, 1974). If $L$ is the maximum value of the likelihood function for a model described by $k$ different parameters, then AIC $=-2 \ln L+2 k$. Smaller values of the AIC indicate a better fit.

The Earth data set contains significantly smaller caldera areas compared to the other planetary bodies considered. Because of erosion processes and vegetation on Earth, measuring and locating small features can be a difficult task and can lead to the underreporting of small calderas. We also observed a divergence of the data from the collapse for the small caldera areas. For these reasons, we performed the goodness of fit test for calderas/paterae with areas larger than $30 \mathrm{~km}^{2}$. This cutoff only affects the Earth and Io data sets. The results of this test are given in Tables 2 and 3.

The obtained AIC values suggest that the GEV distribution provides the best fit for the collapsed data:

$f(y)=\frac{1}{\sigma} u(y)^{-1-1 / \theta} \exp \left[-u(y)^{-1 / \theta}\right]$,

where $u(y)=1+\theta(y-\mu) / \sigma$ with the shape parameter $\theta=$ $0.22 \pm 0.06$, the location parameter $\mu=0.64 \pm 0.04$, and the scale parameter $\sigma=0.42 \pm 0.03$ for the diameters (Fig. 2a) and $\theta=0.84 \pm 0.11, \mu=0.26 \pm 0.03, \sigma=0.30 \pm 0.03$ for the areas (Fig. 2b). The estimates of the parameters were calculated with a $95 \%$ confidence interval. The value of the shape 
Table 3. The maximum values of the $\log$-likelihood function $(\ln L)$ and the corresponding values of the AIC for the model distribution functions considered to fit (a) the rescaled distributions given in Fig. $2 \mathrm{~b}$ and (b) the rescaled distributions given in Fig. $4 . k$ is the number of parameters for the considered distribution. The values correspond to the fitting analysis considering only calderas/paterae with an area above $30 \mathrm{~km}^{2}$.

\begin{tabular}{lccccc}
\hline Distribution & $k$ & (a) $\ln L$ & AIC & (b) $\ln L$ & AIC \\
\hline Generalized Pareto & 2 & -462.0 & 928.1 & -213.8 & 431.6 \\
Log-normal & 2 & -442.4 & 888.8 & -192.6 & 389.2 \\
Generalized Extreme Value & 3 & -435.4 & 876.9 & -185.4 & 376.9 \\
\hline
\end{tabular}

parameter for the GEV distribution characterizes the tail of the distribution: if $\theta>0$, the distribution displays heavy tail behavior as opposed to a light tail behavior when $\theta=0$ or a bounded tail if $\theta<0$. Here, both distributions for the diameters and the areas are heavy tail distributions. The mean value of the GEV distribution is defined as $\mu+\sigma\left[\frac{\Gamma(1-\theta)-1}{\theta}\right]$ and is equal to 1 for both distributions, by definition, because of the normalization process. The standard deviation is defined as $\sqrt{\frac{\sigma^{2}\left(g_{2}-g_{1}^{2}\right)}{\theta^{2}}}$, where $g_{k}=\Gamma(1-k \theta)$ and is equal to 0.81 for the distribution of diameters and is undefined for the distribution of areas since $\theta>0.5$. We observed a deviation from the data collapse for small values of diameters and areas. This discrepancy can be explained by the small number of data in the Martian catalog and by a possible misinterpretation of the smaller landforms for Io. Another explanation for this deviation from the collapse could be a different scaling form that applies to small caldera areas. It is important to note that a similar type of distribution displaying a power-law tail and an exponential rollover was used to fit the distributions of three different landslides inventories (Malamud et al., 2004).

\subsection{The Earth}

We also investigated the scaling properties of caldera sizes on Earth according to their surrounding crustal properties. The CCDB provides size data for calderas worldwide as well as other characteristics such as plate tectonic settings, crustal type, world region, and rock type (Geyer and Marti, 2008). For our analysis, we grouped the calderas according to 3 distinct crustal types: continental silicic crust, oceanic basaltic crust, and transitional crust (see Table 1).

Using the same methodology, we computed the probability density distributions for each of the 3 data sets (Fig. 3). We performed the scaling analysis using the mean caldera area as a scaling factor for each distribution (Fig. 4). Comparably to planetary calderas, we observed a good collapse of all the distributions into a single functional form. Similarly to planetary calderas, we only focused on areas larger than $30 \mathrm{~km}^{2}$. After testing the generalized Pareto distribution, the log-normal distribution and the GEV distribution as fits for

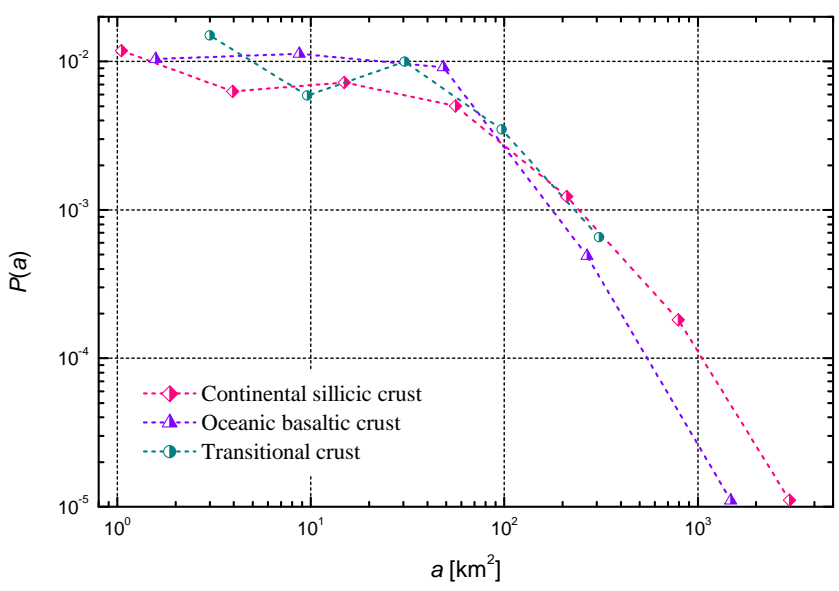

Fig. 3. Probability density functions of caldera areas for three separate crustal types on Earth.

the rescaled distributions (see Table 3), we found that the best AIC value was obtained for the GEV distribution, Eq. (2), with $\theta=0.94 \pm 0.16, \mu=0.22 \pm 0.04$, and $\sigma=0.28 \pm 0.05$. The estimates of the parameters were calculated with a $95 \%$ confidence interval. We note that for this scaling analysis and for the one using the planetary data sets, the GEV parameters are similar within the error limits. We observe a departure from the collapse for small values in the case of the Earth calderas, which could be explained by incompleteness of the catalog or by a different scaling form for small calderas.

\section{Terrestrial volcanic eruption analysis}

Caldera formation is the result of volcanic eruption events. In order to investigate the temporal patterns and characteristic time scales associated with eruptions of all types of volcanoes, we studied the eruption time series of volcanoes around the world. The data were obtained from the Smithsonian Institution global volcanism database (Siebert and Simkin, 2002).

The time interval between two consecutive eruptions, also named interevent time, is an important feature of volcano dynamics (Marzocchi and Zaccarelli, 2006; Deligne et al., 2010). For this study, we considered the onset of an eruption as the time of the arrival of volcanic products at the Earth's surface. This includes explosive ejection of fragmental material or effusion of previously liquid lava.

In order to use data sets with a large enough number of samples, we selected the individual eruption sequences of 13 individual volcanoes located around the world with a large eruptive history: Etna in Italy, Marapi, Merapi and Semeru in Indonesia, Villarrica in Chile, Piton de la Fournaise on the Reunion island, Mauna Loa and Kilauea in Hawaii, Ambrym on Vanuatu, Asama and Aso in Japan, Grimsvotn in Iceland, and Taupo in New Zealand. To increase statistics 


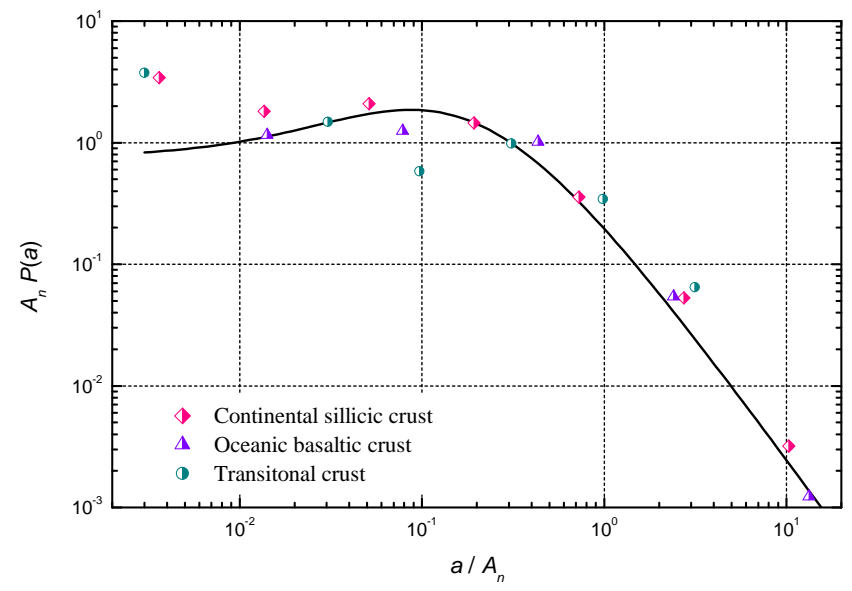

Fig. 4. Rescaled caldera areas distributions for the three crustal types according to Eq. (1) with the mean area $A_{n}$ computed for each crustal type (Table 1). The solid curve is a fit to the rescaled distributions and is given by the GEV distribution, Eq. (2), with $\theta=0.94 \pm 0.16, \mu=0.22 \pm 0.04$, and $\sigma=0.28 \pm 0.05$.

by assuming that volcanoes located in the same region and surrounded by similar tectonic settings produced statistically similar eruption sequences (Rodado et al., 2011), we also used data from less active volcanoes that we grouped into different regional catalogs: Alaska (4 volcanoes considered, 74 eruptions), Aleutians (17 volcanoes, 143 eruptions), Central America (25 volcanoes, 409 eruptions), Iceland (7 volcanoes, 100 eruptions), Indonesia (39 volcanoes, 771 eruptions), Italy (3 volcanoes, 187 eruptions), Japan (22 volcanoes, 487 eruptions), Kamchatka (13 volcanoes, 293 eruptions), New Zealand (4 volcanoes, 175 eruptions), and South America (36 volcanoes, 518 eruptions). A more detailed analysis of the temporal scaling properties of volcanism on Earth can be found in Sanchez and Shcherbakov (2012).

For each volcano eruption sequence, we computed the time intervals $\Delta t$ between successive eruptions as $\Delta t_{i}=$ $t_{i}-t_{i-1}$, with $t_{i}$ being the time onset of the $i$-th eruption. For the analysis, we ignored the eruption duration but instead measured the interevent time between the onset of one eruption to the onset of the subsequent eruption. The eruption duration can vary for one volcano from a day to several years. For the oldest eruptions, the duration may not even be reported (Siebert and Simkin, 2002). It is for these reasons that we decided not to consider the duration of the events for this analysis. To ensure the completeness of the catalogs considered, we applied a date cutoff to each individual volcano by detecting when the data became less reliable based on the number of uncertain dates and changes in the mean rate of eruptions. This method might not fully account for uncertain or missing eruption dates, but at present there is no truly reliable technique to validate the completeness of the volcanic eruption catalogs to ensure sufficient data for the analysis. For each of the catalogs considered, when the day of the event was not specified, we assigned it as being the first day of the month. When both the day and the month were missing, we assigned the date to the first of July.

To represent the tail of the distribution of interevent times, we also compiled a group composed of eruption sequences of 16 volcanoes located around the world and characterized by the presence of a caldera and long interevent times between eruptions. This was done assuming that the processes controlling the eruptions of this type of volcanoes were similar and therefore their interevent time distributions were comparable. For the caldera data sets, we did not introduce a date cutoff since the eruptions characterizing these volcanoes were all of considerable size and present in the geological records. We therefore assumed that the eruptive histories were approximately complete.

Similarly to calderas, because multiple time scales were involved (days to hundreds of years), we used logarithmically increasing bin lengths to plot the probability densities. The data sets for grouped volcanoes belonging to specific regions typically consist of several volcanoes. Each volcano has its own rate of eruption. For processes with the exponential trend in the distribution of interevent times, it is appropriate to rescale first the interevent times of each individual volcano sequence with the corresponding sample mean value and then combine those data sets into a single one (Marzocchi and Zaccarelli, 2006). This effectively transforms each volcano interevent sequence into one with a unit rate.

For each individual volcano eruption sequence belonging to the 11 groups (10 volcanically active regions and a separate caldera group), we computed the corresponding interevent times and the sample mean $\tau_{n}$. We then divided each interevent time $\Delta t_{i}$, by this sample mean in order to normalize each interevent sequence to have the same unit mean rate. After that we combined these normalized interevent times from all volcanoes belonging to a specific region and computed their interevent time distribution functions. This was done to ensure that the distributions were computed from the data having the same rate of occurrence (Marzocchi and Zaccarelli, 2006). This approach is essentially the same as given by Eq. (1). We also performed the scaling analysis on the individual volcanoes following Eq. (1). The result of this rescaling is shown in Fig. 5. We observe a good collapse of all the distributions into a single functional form.

To fit the scaling function $f(y)$, we tested the same 3 heavy-tailed distributions used for the caldera analysis (the generalized Pareto distribution, the log-normal distribution and the GEV distribution), as well as the gamma and the exponential distributions, which are both part of the exponential family and a common choice when fitting interevent time distributions. The AIC values for each of the distributions are shown in Table 4. From this analysis, we concluded that the log-normal distribution fitted the data best: 


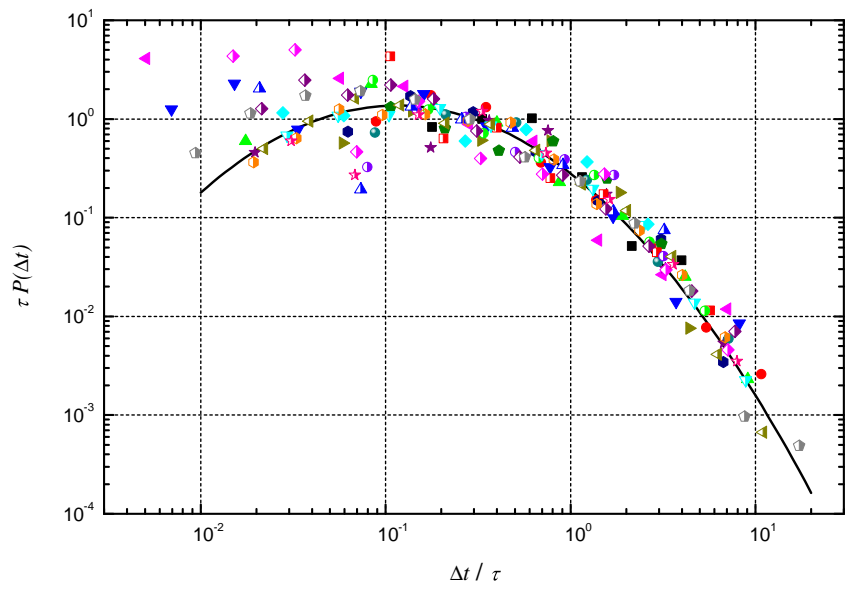

Fig. 5. Rescaled interevent time distributions for the individual volcanoes and groups of volcanoes according to Eq. (1), with the mean interevent time $\tau_{n}$ computed for each distribution. The solid curve is a fit to the rescaled distributions and is given by the log-normal distribution, Eq. (3), with $\mu=-0.69 \pm 0.04$ and $\sigma=1.21 \pm 0.03$.

$f(y)=\frac{1}{y \sigma \sqrt{2 \pi}} \exp \left\{-\frac{[\ln (y)-\mu]^{2}}{2 \sigma^{2}}\right\}$,

with $\mu=-0.69 \pm 0.04$ and $\sigma=1.21 \pm 0.03$. This distribution has already been reported to describe the conditional probability density function of the times to the next eruption given a magnitude of the current eruption for open conduit systems (Marzocchi and Zaccarelli, 2006).

\section{Conclusions}

Calderas are formed by the collapse of the edifice of the erupting volcanoes into partially drained magma chambers. Their sizes are related to the complex processes that build the original volcanoes, to the propagation of the rising magma through the host crustal rock, and to the size of the magma chamber itself. Therefore, the caldera size distribution is an important characteristics that can be used to infer and constrain the geodynamical processes operating on planetary bodies.

Using the proposed scaling analysis, we have shown that the distributions of caldera diameters and areas on Earth, Mars, Venus and Io can be described as a universal function, the Generalized Extreme Value (GEV) distribution. We also analyzed the distribution of caldera sizes on Earth by grouping them into 3 distinct crustal types: continental silicic crust, oceanic basaltic crust, and transitional crust. We showed that their distributions were described by the same GEV distribution. The obtained parameters of the GEV distribution for planetary calderas and for the calderas on Earth with distinct crustal types were similar within error limits. This inferred
Table 4. The maximum values of the $\log$-likelihood function $(\ln L)$ and the corresponding values of the AIC for the model distribution functions considered to fit the rescaled distributions given in Fig. 5. $k$ is the number of parameters for the considered distribution.

\begin{tabular}{lccc}
\hline Distribution & $k$ & $\ln L$ & AIC \\
\hline Generalized Pareto & 2 & -3586.9 & 7177.9 \\
Log-normal & 2 & -3493.2 & 6990.5 \\
Generalized Extreme Value & 3 & -3579.1 & 7164.3 \\
Gamma & 2 & -3792.0 & 7586.0 \\
Exponential & 1 & -3761.0 & 7525.9 \\
\hline
\end{tabular}

scaling implies that the dynamic processes responsible for caldera formation are similar for the Earth, Mars, Venus, and Io and are quite independent of the crustal settings on Earth. The mean caldera/patera diameters and areas play the role of characteristic sizes for each of the distributions. This result has important implications for physical constraints on any realistic model describing the volcano formation and eruption. In this context, the comparative planetology is a powerful approach that can help to further understand caldera formation in the solar system.

By investigating the temporal behavior of the eruption of terrestrial volcanoes, we aimed at constraining stochastic properties of volcanic eruptions. These eruptions are, in most cases, characterized by non-trivial temporal correlations. The scaling analysis of the distribution of interevent times between successive eruptions performed on individual volcanoes and volcanic groups located around the world and surrounded by various tectonic settings lead to the collapse of these distributions into a single functional form that we modeled using the log-normal distribution. The obtained scaling implies that the distributions are controlled by the mean interevent time, which plays the role of a characteristic time scale. The log-normal temporal behavior of volcanic eruptions allows us to conclude that the temporal structure of volcanic sequences deviates from the simple Poisson statistics. By investigating the scaling properties of volcanic eruptions, we aimed at finding similarities in the temporal behavior of eruptions on Earth. We used a large number of volcanoes located around the world and surrounded by different tectonic settings. The data collapse observed lead us to conclude that the temporal behavior of those volcanoes displays significant similarities.

Acknowledgements. This work has been supported by NSERC Discovery grant $355632-2008$. We are grateful to the two anonymous referees for their helpful suggestions.

Edited by: S. Lovejoy

Reviewed by: two anonymous referees 


\section{References}

Acocella, V.: Understanding caldera structure and development: An overview of analogue models compared to natural calderas, Earth. Sci. Rev., 85, 125-160, 2007.

Akaike, H.: A new look at the statistical model identification, IEEE Trans. Autom. Contr., 19, 716-723, 1974.

Baiesi, M., Paczuski, M., and Stella, A.: Intensity threshold and the statistics of the temporal occurence of solar flares, Phys. Rev. Lett., 96, 051 103, 2006.

Bebbington, M. S.: Trends and clustering in the onsets of volcanic eruptions, J. Geophys. Res., 115, B01203,doi:10.1029/2009JB006581, 2010

Bebbington, M. S. and Lai, C. D.: On nonhomogeneous models for volcanic eruptions, Math. Geol., 28, 585-600, 1996.

Bulmer, M. H. and Wilson, J. B.: Comparison of flat-topped stellate seamounts on Earth's seafloor with stellate domes on Venus using side-scan sonar and Magellan synthetic aperture radar, Earth Planet. Sci. Lett., 171, 277-287, 1999.

Corral, Á.: Local distributions and rate fluctuations in a unified scaling law for earthquakes, Phys. Rev. E, 68, 035102, doi:10.1103/PhysRevE.68.035102, 2003.

Corral, Á., Telesca, L., and Lasaponara, R.: Scaling and correlations in the dynamics of forest-fire occurence, Phys. Rev. E, 77, 016101, doi:10.1103/PhysRevE.77.016101, 2008.

Corral, Á., Ossó, A., and Llebot, J.: Scaling of tropical-cyclone dissipation, Nat. Phys., 6, 693-696, 2010.

Crumpler, L. S., Aubele, J. C., and Head III, J. W.: The Tabulated Magellan Venus Volcanic Feature Catalog, available at: http://www.planetary.brown.edu/planetary/databases/venus _ cat.html (last access: April 2012), 1997.

Davidsen, J., Stanchits, S., and Dresen, G.: Scaling and universality in rock fracture, Phys. Rev. Lett., 98, 125502, doi:10.1103/PhysRevLett.98.125502, 2007.

De la Cruz-Reyna, S.: Poisson-distributed patterns of explosive eruptive activity, B. Volcanol., 54, 57-67, doi:10.1007/BF00278206, 1991.

Deligne, N. I., Coles, S. G., and Sparks, R. S. J.: Recurrence rates of large explosive volcanic eruptions, J. Geophys. Res., 115, B06203, doi:10.1029/2009JB006554, 2010.

Frey, H. and Jarosewich, M.: Subkilometer Martian volcanoes: Properties and possible terrestrial analogs, J. Geophys. Res., 87, 9867-9879, 1982.

Geyer, A. and Marti, J.: The new worldwide collapse caldera database (CCDB): A tool for studying and understanding caldera processes, J. Volcanol. Geotherm. Res., 175, 334-354, doi:10.1016/j.jvolgeores.2008.03.017, 2008.

Gusev, A. A.: Temporal structure of the global sequence of volcanic eruptions: Order clustering and intermittent discharge rate, Phys. Earth Planet. Inter., 166, 203-218, doi:10.1016/j.pepi.2008.01.004, 2008.

Gusev, A. A., Ponomareva, V., Braitseva, O. A., Melekestsev, I. V., and Sulerzhitsky, L. D.: Great explosive eruptions on Kamchatka during the last 10,000 years: Self-similar irregularity of the output of volcanic products, J. Geophys. Res., 108, 2126, doi:10.1029/2001JB000312, 2003.

Ho, C. H.: Non homogeneous Poisson model for volcanic eruptions, Math. Geol., 23, 167-173, 1991.
Hughes, G. and Mahood, G.: Silicic calderas in arc settings: Characteristics, distribution, and tectonic controls, Geol. Soc. Am. Bull., 123, 1577-1595, 2011.

Kieffer, S. W.: Numerical models of caldera-scale volcanic eruptions on Earth, Venus, and Mars, Science, 269, 1385-1391, 1995.

Lipman, P. W.: Calderas, in: Encyclopedia of Volcanoes, edited by: Sigurdsson, H., Houghton, B. F., McNutt, S. R., Rymer, H., and Stix, J., Academic Press, San Fransisco, 643-662, 2000.

Malamud, B., Turcotte, D., Guzzetti, F., and Reichenbach, P.: Landslide inventories and their statistical properties, Earth Surf. Proc. Land., 29, 687-711, 2004.

Marzocchi, W. and Zaccarelli, L.: A quantitative model for the timesize distribution of eruptions, J. Geophys. Res., 111, B04204, doi:10.1029/2005JB003709, 2006.

Mendoza-Rosas, A. T. and De la Cruz-Reyna, S.: A mixture of exponentials distribution for a simple and precise assessment of the volcanic hazard, Nat. Hazards Earth Syst. Sci., 9, 425-431, doi:10.5194/nhess-9-425-2009, 2009.

Mouginis-Mark, P. and Rowland, S.: The geomorphology of planetary calderas, Geomorphology, 37, 201-223, 2001.

Prockter, L., Ernst, C., Denevi, B., Chapman, C., Head III, J., Fassett, C., Merline, W., Solomon, S., Watters, T., Strom, R., Cremonese, G., Marchi, S., Massironi, M.: Evidence for young volcanism on Mercury from the third MESSENGER flyby, Science, 329, 668-671, 2010.

Pyle, D. M.: Forecasting sizes and repose times of future extreme volcanic events, Geology, 26, 367-370, 1998.

Radebaugh, J., Keszthelyi, L. P., McEwen, A. S., Turtle, E. P., Jaeger, W., and Milazzo, M.: Paterae on Io: A new type of volcanic caldera?, J. Geophys. Res., 106, 33005-33020, 2001.

Rodado, A., Bebbington, M., Noble, A., Cronin, S., and Jolly, G.: On Selection of Analog Volcanoes, Math. Geosci., 43, 1-15, 2011.

Salvi, F., Scandone, R., and Palma, C.: Statistical analysis of the historical activity of Mount Etna, aimed at the evaluation of volcanic hazard, J. Volcanol. Geotherm. Res., 154, 159-168, 2006.

Sanchez, L. and Shcherbakov, R.: Temporal Scaling of Volcanic Eruptions, J. Volcanol. Geotherm. Res., 247-248, 115-121, doi:10.1016/j.jvolgeores.2012.08.004, 2012.

Shcherbakov, R., Yakovlev, G., Turcotte, D. L., and Rundle, J. B.: Model for the Distribution of Aftershock Interoccurrence Times, Phys. Rev. Lett., 95, 218501, doi:10.1103/PhysRevLett.95.218501, 2005.

Siebert, L. and Simkin, T.: Volcanoes of the World: An Illustrated Catalog of Holocene Volcanoes and their Eruptions, Smithsonian Institution, Global Volcanism Program Digital Information Series, GVP-3, available at: http://www.volcano.si.edu/world/ (last access: June 2012), 2002.

Sigurdsson, H., Houghton, B. F., McNutt, S. R., Rymer, H., and Stix, J.: Encyclopedia of Volcanoes, Academic Press, New York, 2000.

Smith, D. K.: Comparison of the shapes and sizes of seafloor volcanoes on Earth and "pancake" domes on Venus, J. Volcanol. Geotherm. Res., 73, 47-64, 1996.

Sobradelo, R., Geyer, A., and Martí, J.: Statistical data analysis of the CCDB (Collapse Caldera Database): Insights on the formation of caldera systems, J. Volcanol. Geotherm. Res., 198, 241$252,2010$. 
USGS: USGS astrogeological program, available at: http://planetarynames.wr.usgs.gov/, last access: April 2012.

Wickman, F. E.: Repose period patterns of volcanoes. I. Volcanic eruptions regarded as random phenomena, Ark. Kem. Mineral. Geol., 4, 291-302, 1966.
Wilson, L.: Volcanism in the Solar System, Nat. Geosci., 2, 388396, doi:10.1038/ngeo529, 2009.

Wood, C. A.: Calderas: A Planetary Perspective, J. Geophys. Res., 89, 8391-8406, 1984. 